\title{
Search for tidal dwarf galaxy candidates in a sample of ultraluminous infrared galaxies
}

\author{
A. Monreal-Ibero ${ }^{1}$, L. Colina ${ }^{2}$, S. Arribas ${ }^{2}$, and M. García-Marín ${ }^{2}$ \\ 1 Astrophysikalisches Institut Potsdam, An der Sternwarte 16, 14482 Potsdam, Germany \\ e-mail: amonreal@aip.de \\ 2 Instituto de Estructura de la Materia (CSIC), Serrano 121, 28006 Madrid, Spain \\ e-mail: [colina;arribas;maca]@damir.iem.csic.es
}

Received 17 November 2006 / Accepted 28 May 2007

ABSTRACT

\begin{abstract}
Context. Small star-forming galaxies made out of collisional debris have been found in a variety of merging systems. So far only a few of them are known in Ultraluminous Infrared Galaxies (ULIRGs) although they show clear signs of interactions. Whether external star formation may take place in such objects in an open question.

Aims. The aim of this paper is to identify and characterise the physical and kinematic properties of the external star-forming regions in a sample of ULIRGs, including Tidal Dwarf Galaxies (TDG) candidates. The likelihood of survival of these regions as TDGs is also evaluated.

Methods. The analysis is based on optical Integral Field Spectroscopy (IFS) and high angular resolution Hubble Space Telescope (HST) imaging.

Results. We found that the presence of external star-forming regions is common, with 12 objects being identified in 5 ULIRGs. These regions show a large range of dynamical mass up to $1 \times 10^{10} M_{\odot}$, with average sizes of $\sim 750 \mathrm{pc}$. In addition, the line ratios (H II region-like), metallicities $(12+\log (\mathrm{O} / \mathrm{H}) \sim 8.6)$ and $\mathrm{H} \alpha$ equivalent widths (34-257 $\mathrm{A}$ ) are typical of young bursts of star formation (age 5-8 Myr), and similar to those of the TDG candidates found in less luminous mergers and compact groups of galaxies. The extinction corrected $\mathrm{H} \alpha$ luminosity of these young bursts leads to masses for the young stellar component of $\sim 2 \times 10^{6}-7 \times 10^{8} M_{\odot}$. The likelihood of survival of these regions as TDGs is discussed based on their structural and kinematic properties. Particularly interesting is our finding that most of these systems follow the relation between effective radius and velocity dispersion found at lower (globular clusters) and higher (Elliptical) mass systems, which suggests they are stable against internal motions. The stability against forces from the parent galaxy is studied on the basis of several criteria and a comparison of the data with the predictions of dynamical evolutionary models is also performed. Five regions out of twelve show High-Medium or High likelihood of survival based on all the utilised tracers. Our best candidate, which satisfies all criteria, is located in the advanced merger IRAS $15250+3609$ and presents a velocity field decoupled from the relatively distant parent galaxy.
\end{abstract}

Key words. galaxies: active - galaxies: interactions - galaxies: starburst - galaxies: dwarf

\section{Introduction}

Ultraluminous Infrared Galaxies (ULIRGs) are defined as objects with an infrared luminosity $L(8-1000 \mu \mathrm{m}) \gtrsim 10^{12} L_{\odot}($ e.g. Sanders \& Mirabel 1996). This huge luminosity is believed to be caused mainly by star formation, although the existence of an Active Galactic Nucleus (AGN) cannot be discarded and could be the dominant source of energy in a small percentage of these systems (for reviews see Sanders \& Mirabel 1996; Lonsdale et al. 2006). ULIRGs are systems rich in gas and dust (e.g. Evans et al. 2002; Evans et al. 2000) and all of them present emission lines in the optical (e.g. Veilleux et al. 1999; Kim et al. 1998). Observational studies about the morphology of these systems indicate that most (if not all) of them show signs of mergers and interaction (e.g. Clements et al. 1996; Borne et al. 2000; Bushouse et al. 2002; Cui et al. 2001; Veilleux et al. 2002) while theoretical simulations of mergers indicate that ULIRGs constitute a welldefined subgroup within mergers in general. As such, in principle only major mergers of two gas-rich spiral galaxies are able to explain the large luminosity observed in ULIRGs and only during a short period of time ( $\sim 50 \mathrm{Myr}$, e.g. Mihos \& Hernquist 1996; Bekki \& Shioya 2001). In this context, there are some empirical evidences suggesting that ULIRGs are mergers of two or more (sub)- $L^{*}$ spirals (Colina et al. 2001), which could be the progenitors of intermediate-mass ellipticals (Genzel et al. 2001; Tacconi et al. 2002). ULIRGs could also evolve into quasars (Sanders et al. 1988), at least in those mergers where the progenitor galaxies are more massive (Colina et al. 2001).

One issue regarding ULIRGs that deserves attention is the presence of knots and condensations of star formation outside the nuclei. On the one hand, at the lowest masses and smallest sizes range, we find the so-called Super Stellar Clusters (e.g. Scoville et al. 2000; Shioya et al. 2001; Surace \& Sanders 2000, 1999). These systems, with typical masses in the range of $10^{4}-$ $10^{6} M_{\odot}$ and sizes of $r \sim 5 \mathrm{pc}$, are believed to be the progenitors of current globular clusters (e.g. Schweizer et al. 1996). In addition to ULIRGs, they have been found in a wide range of environments such as starburst galaxies (e.g. Meurer et al. 1995; Melo et al. 2005), the space between galaxies in compact groups (Gallagher et al. 2001; Mendes de Oliveira et al. 2004) and especially in mergers less luminous than those studied here (e.g. Zepf et al. 1999; Whitmore et al. 1999; Alonso-Herrero et al. 2002; Knierman et al. 2003; Bastian et al. 2006). On the other hand, at masses $\sim 10^{8}-10^{9} M_{\odot}$ we find the so-called Tidal Dwarf Galaxies (TDG). Their existence was proposed in the 50's when Zwicky (1956) suggested that the tidal forces in interacting galaxies could create self-gravitating objects made up from 
the debris of the interaction, which could become small galaxies. From the observational point of view, these kind of objects, with masses and sizes similar to dwarf galaxies and large quantities of gas (e.g. Braine et al. 2001) have already been found in less luminous interacting or merging galaxies (Duc \& Mirabel 1994, 1998; Duc et al. 2000; Hibbard et al. 2001; Weilbacher et al. 2000; Weilbacher et al. 2003) or in compact groups of galaxies (Iglesias-Páramo \& Vílchez 2001; Mendes de Oliveira et al. 2001; Temporin et al. 2003; López-Sánchez et al. 2004; Amram et al. 2004; Lisenfeld et al. 2004) while modelling has shown that the formation of condensations is possible in mergers between two disc galaxies, as occurs in ULIRGs (e.g. Duc et al. 2004; Wetzstein et al. 2005; Bournaud \& Duc 2006).

In spite of the fact that they have the appropriate nature to harbour TDG candidates, no systematic search for these candidates among ULIRGs has been performed to date. The TDG candidate in The Superantennae (Mirabel et al. 1991), turned out to be a background object and to our knowledge, only three more candidates have been identified so far (Mihos \& Bothun 1998).

An interesting question is whether these objects are likely to contain the same quantity or more TDGs than other types of merging systems. More generally, are ULIRGs favourable sites for star formation in and extended mode? Regarding gas distribution, it seems that ULIRGs are particularly efficient in driving gas to the innermost regions, meaning that there is, a priori, much smaller gas reservoir in the outer parts. However, detailed simulations able to explain the luminosities observed in these objects and focused in the gas distribution, show a dual behaviour for the gas: while that in the inner disc (typically $\sim 5 \mathrm{kpc}$ ) flows directly toward the central regions within $1-2 \times 10^{8} \mathrm{yr}$ after the pericentric passage, the outer gas is ejected into tidal tails and bridges (Iono et al. 2004). Regarding the extent of star formation in mergers, Barnes (2004) showed that this is larger when shockinduced star formation laws are used instead of local gas density based laws. From the observational point of view, the fact that ULIRGs are more gas-rich than less luminous interacting systems and the presence of shocks in these systems (Monreal-Ibero et al. 2006) may favour extended star-formation. In this context, the positive or negative detection of TDGs (or external star formation in general) may help to clarify these issues.

Finally, quantifying the incidence of TDGs in local ULIRGs may have cosmological implications since these objects are thought to be the local counterpart of the so-called Spitzer and sub-millimeter sources (e.g. Smail et al. 1997; Hughes et al. 1998) at $z \sim 1-2$. These sources present similar or even greater luminosities than ULIRGs (see Blain et al. 2002, for a review of their properties) and a relatively high fraction have morphological properties consistent with being systems suffering an interaction or merging process (Chapman et al. 2003; Pope et al. 2005; Smail et al. 2004; Ivison et al. 2000; Conselice et al. 2003). In addition, they are two orders of magnitude more numerous than local ULIRGs (Pérez-González et al. 2005; Caputi et al. 2007). Thus, while the present-day ULIRGs could provide a relatively small contribution to the total number of newly created TDGs, their high- $z$ counterpart might be fundamental in this regard. Hence detection of this kind of regions among ULIRGs may increase considerably the estimation of the percentage of current dwarfs that could have been formed from tidal debris.

In this paper, we characterise the extranuclear star forming regions of a sample of ULIRGs using the combined information of Integral Field Spectroscopy (IFS) data together with high resolution images from the HST. Properties such as extinction, ionisation state, metallicity, age and mass of the stellar population, velocity dispersion, relative velocity etc., are derived. We use these parameters to estimate the likeliness of survival of these regions as future TDGs. The present study is part of a wider program whose final aim is to perform a detailed study of a representative sample of ULIRGs using IFS. Due to the complex nature of these systems, this technique, that allows simultaneous identification of spectral and spatial information, is well suited for their characterisation. Previous results of this program can be found in Monreal-Ibero et al. (2006) and references therein.

Throughout the paper, a cosmology with $70 \mathrm{~km} \mathrm{~s}^{-1} \mathrm{Mpc}^{-1}$, $\Omega_{\mathrm{M}}=0.3$ and $\Omega_{\Lambda}=0.7$ is assumed.

\section{Sample and observations}

\subsection{Sample}

The galaxies studied were selected from a sample of nine ULIRGs with IFS data, including those presented in Colina et al. (2005) plus IRAS 16007+3743 (García-Marín 2005). They cover the ULIRGs low luminosity range $\left(12.10 \leq \log \left(L_{\mathrm{IR}} / L_{\odot}\right) \leq\right.$ $12.60)$, have a variety of activity classes, and are in different stages of the interaction process.

Potential TDG candidates have been selected as any high surface brightness compact region in the emission line maps (obtained from the IFS data) at a distance from the nucleus of the galaxy larger than 2 .'0, and associated with a single condensation in the WFPC2 image.

Following the above selection criteria, no regions are found in Mrk 273 (Colina et al. 1999), Arp220 (Arribas et al. 2001) or IRAS 17208-0014 (Arribas \& Colina 2003) in the IFS data. IRAS $15206+3342$ presents a chain of knots at a projected distance of about 3.5-7.5 kpc from the nucleus in the WFPC2 image (Arribas \& Colina 2002). However, the spatial resolution of the IFS data does not allow us to derive their individual properties and, therefore, this system is not be considered here.

Thus, the final sample includes the following five systems with at least one region of interest: IRAS $08572+3915$, IRAS 12112+0305, IRAS 14348-1447, IRAS 15250+3609 and IRAS $16007+3743$. IRAS $15250+3609$ is an advanced merger while the rest, with two nuclei and a more spread star formation, are in an earlier stage of the merging process. Table 1 shows some relevant properties of the selected galaxies. With the exception of IRAS $16007+3743$, which is at a slightly higher redshift, typical redshifts for these systems are $z \sim 0.07$ which implies a linear scale of about $1.3 \mathrm{kpc} \operatorname{arcsec}^{-1}$.

\subsection{Observations}

The IFS data were obtained with the INTEGRAL system (Arribas et al. 1998) plus the WYFFOS spectrograph (Bingham et al. 1994) in the $4.2 \mathrm{~m} \mathrm{WHT}$ at the Observatorio del Roque de los Muchachos (Canary Islands) on April 1998 and April 2001. Spectra were taken using a 600 lines $\mathrm{mm}^{-1}$ grating with an effective resolution of $4.8 \AA$. We used the fibre bundle SB2 which has a field of view of $16^{\prime \prime} .0 \times 12^{\prime \prime} .3$ and is made up of 219 fibres, each 0.45 in radius. Fibres are arranged in two sets which observe simultaneously the target and the sky. The covered spectral range, exposure time and air mass for each object can be found in Monreal-Ibero et al. (2006). The data for IRAS 16007+3743 were obtained with a similar configuration and a total exposure time of $6 \times 1500 \mathrm{~s}$. 
Table 1. ULIRGs sample.

\begin{tabular}{ccccccc}
\hline \hline Galaxy & $z^{a}$ & $\begin{array}{c}\text { Scale } \\
\left(\mathrm{kpc} \mathrm{arcsec}^{-1}\right)\end{array}$ & $\log \left(L_{\mathrm{IR}} / L_{\odot}\right)^{b}$ & IR Class $^{c}$ & $\begin{array}{c}\text { Interaction } \\
\text { class }^{d}\end{array}$ & Regions $^{e}$ \\
\hline IRAS 08572+3915 & 0.058 & 1.13 & 12.17 & $\mathrm{~W}$ & III & 2 \\
IRAS 12112+0305 & 0.073 & 1.39 & 12.37 & $\mathrm{C}$ & III & 5 \\
IRAS 14348-1447 & 0.083 & 1.56 & 12.40 & $\mathrm{C}$ & III & 1 \\
IRAS 15250+3609 & 0.055 & 1.07 & 12.09 & $\mathrm{C}$ & IV & 1 \\
IRAS 16007+3743 & 0.185 & 3.10 & 12.11 & $\mathrm{C}$ & III & 3 \\
\hline
\end{tabular}

a Redshifts taken from the NASA/IPAC Extragalactic Database (NED).

${ }^{b}$ Infrared luminosities calculated using the infrared IRAS fluxes of Moshir et al. (1993), the $L_{\mathrm{IR}}$ expression given in Sanders \& Mirabel (1996) and a luminosity distance assuming $H_{0}=70 \mathrm{~km} \mathrm{~s}^{-1} \mathrm{Mpc}^{-1}, \Omega_{\mathrm{M}}=0.3$ and $\Omega_{\Lambda}=0.7$.

$c$ Standard IR classification where warm (W) ULIRGs have $f_{25} / f_{60}>0.2$ while cold (C) ULIRGs have $f_{25} / f_{60}<0.2$.

$d$ Following the criterion proposed by Veilleux et al. (2002) where III means pre-merger and IV, merger state.

$e$ Number of external star-forming regions analysed in this work.

\section{Data reduction and analysis}

General reductions for the present IFS data have been discussed somewhere else (e.g. Monreal-Ibero et al. 2006, and references therein). Here we detail the procedure for the absolute calibration of the data, which has not been included in previous papers in this series and is of particular relevance for deriving the $\mathrm{H} \alpha$ luminosity of the regions under study.

\subsection{INTEGRAL data absolute flux calibration}

IFS seems to be a suitable technique to carry out the absolute flux calibration in objects such as ULIRGs, with a complex morphological and kinematical structure and at a certain redshift. Other options involve greater difficulties. For example, observations with long-slit present centring problems, which worsen in the presence of differential atmospheric refraction. On the other hand, observations with narrow filters are complicated to calibrate due to redshift, internal movements of the system and blending of various lines, in particular $\mathrm{H} \alpha+[\mathrm{N} \mathrm{II}]$.

As a first step, the relative flux calibration was carried out using the fibre with greatest signal-to-noise ratio $(\mathrm{S} / \mathrm{N})$ in the calibration star image to create a sensibility function which was used to calibrate every spectrum. After that, we corrected from possible effects of differential atmospheric refraction (Arribas et al. 1999; Filippenko 1982), and the light lost between fibres which affects the calibration star observations. Note that one can infer the fraction of the total flux collected by the selected fibre from images generated from the IFS data at different wavelengths (in a $\Delta \lambda$ ). This information allows us to identify the conversion factors counts/absolute flux and, therefore, calibrate absolutely the IFS data. In practice we proceed as follows. Firstly, we divided the calibration star spectra in several sections of a few Amstrongs each. Secondly, using the flux measured for each fibre, and its position in the bundle, we created a flux map of the calibration star for each section. Finally, we measured in that map the flux collected by the selected fibre and the total flux of the star in arbitrary units. The ratio between the flux collected by the fibre to the total flux of the star in the map allows us to convert the counts from the star into absolute flux in the selected wavelength. This is repeated for a set of images at different wavelengths, providing the conversion curve. In this specific case, we obtained the conversion factor generating images (at $0.04 \operatorname{arcsec}_{\mathrm{pix}^{-1}}$ ) in ten sections of $300 \AA$ each through the entire observed spectral range.

Three tests were performed to estimate the accuracy of this calibration method. Firstly, we compared the total measured flux in a certain spectral range for an image of a star once calibrated with additional calibration stars available for the night. Secondly, the internal consistency of the calibration method was checked by comparing measurements in $\mathrm{H} \alpha$ and $\mathrm{H} \beta$ in certain emission line regions for one of our targets (IRAS 12112+0305) which had been observed on two different nights, pointings and rotation angles. Finally, the $\mathrm{H} \alpha$ flux measured in the nuclei of this system were compared with those found in the literature (Kim et al. 1998) simulating the aperture of a slit in the interpolated maps. Using all these control tests we estimate uncertainties in the flux calibration to be about $10-15 \%$.

\subsection{HST data}

Data archival images from the HST taken with the WFPC2 ( $F 814 W$ filter) were also used to complete our analysis. All images except those of IRAS $08572+3915$ were taken in snapshot mode and with the optional parameter CR-SPLIT activated. For every pointing there were two already reduced images which were combined to reject cosmic rays. Integrated counts for a given aperture were converted to magnitudes in the Vega system using the expressions given in Baggett et al. (2002).

The $F 814 W$ filter is similar to the Johnson-Cousin $I$ filter. Transformations between the WFPC2 and the Johnson-Cousin filter systems need at least measurements in two filters (see for example Origlia \& Leitherer 2000). Given that in most of the regions, the only HST measurement available was that using this filter, we decided not to apply any transformation and to use directly the $F 814 \mathrm{~W}$ magnitudes as if they were $I$ magnitudes. We performed a comparison between the expected magnitudes for the $F 814 W$ and $I$ filter with SYNPHOT using different synthetic spectra. The most relevant for this study was that corresponding to an instantaneous burst of $6 \mathrm{Myr}$ with different levels of extinction. Differences were always 0.1 mag or less.

\section{Results}

\subsection{Identification of the regions of interest}

The selected regions are identified as the bright condensations in the emission lines outside the nuclear region (i.e. at distances of at least $2 \mathrm{kpc}$ from the nucleus). They are marked in the first column of Fig. 1 which contains the WFPC2/HST images. The other two columns in this figure show the continuum and $\mathrm{H} \alpha$ emission line maps. The stellar continuum was obtained averaging two continuum ranges towards the blue and red of the $\mathrm{H} \alpha$ emission line simulating the action of a filter. For the ionised gas 

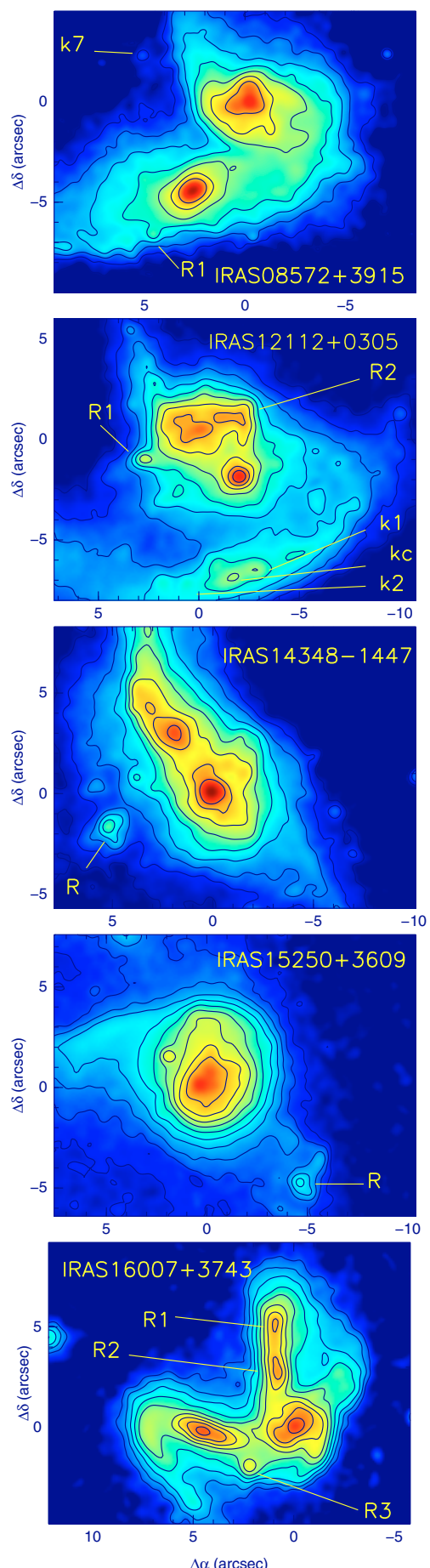
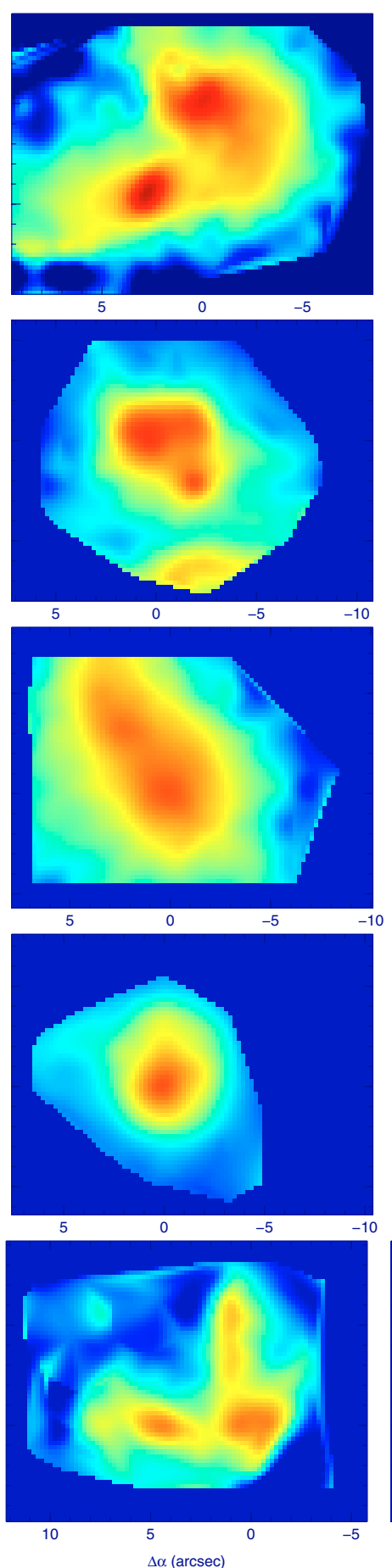
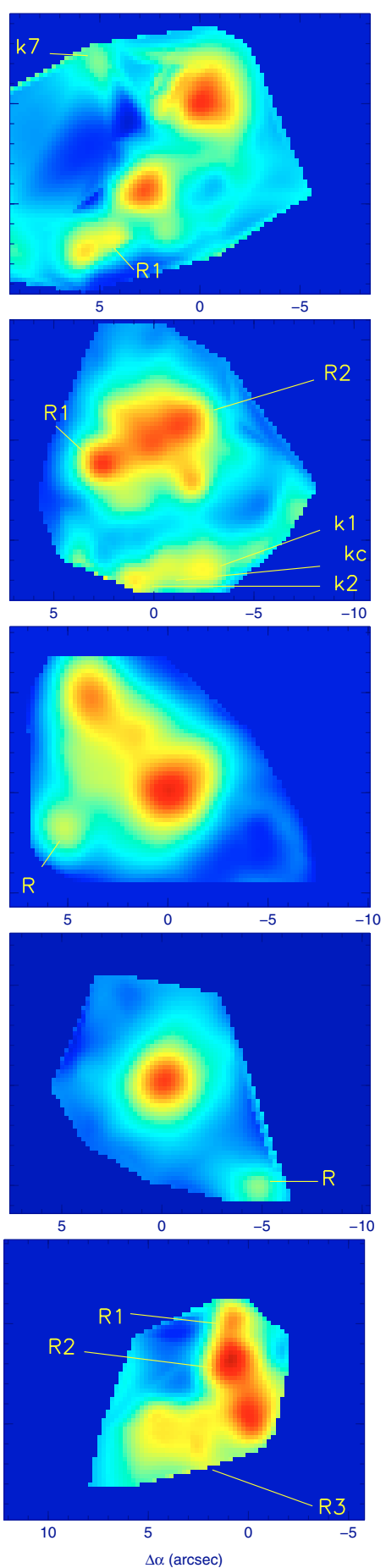

Fig. 1. Sample of ULIRGs used in this study. First column displays the WFPC2 image in the $F 814 W$ filter (i.e. HST $I$-band) for reference. Images have been smoothed with a 0'.2-sigma Gaussian and contours have been over-plotted to better visualise the regions under study. Second and third columns present the maps created from the INTEGRAL data for a line-free continuum adjacent to $\mathrm{H} \alpha$ and for this line respectively. All images are presented in logarithmic scale. North is up and east is to the left. Regions under study have been marked in both the WFPC2 and the INTEGRAL $\mathrm{H} \alpha$ images to better visualise the matching between images from both instruments. (This figure is available in color in electronic form.)

component, we have fitted Gaussian functions to the emission lines using DIPSO package (Howarth \& Murray 1988) within the STARLINK environment (details can be found in MonrealIbero et al. 2006). The general morphology of the stellar and ionised gas component are similar although the stellar component presents a more spread distribution than that of the ionised gas. In the following, we describe briefly the identified regions.
IRAS 08572+3915: Two condensations have been identified here: $\mathrm{k} 7$ at $\sim 6^{\prime \prime}$ from the northern galaxy toward the northeast, near its tidal tail (Arribas et al. 2000) and R1 (k4+5 in the notation of Arribas et al. 2000) in the southern tidal tail and at a distance of $\sim 5^{\prime \prime} .7$ from the southern nucleus. Here $\mathrm{k} 4+5$ will be treated as a single region as we were not able to close independent contours for them in the WFPC2 image. 

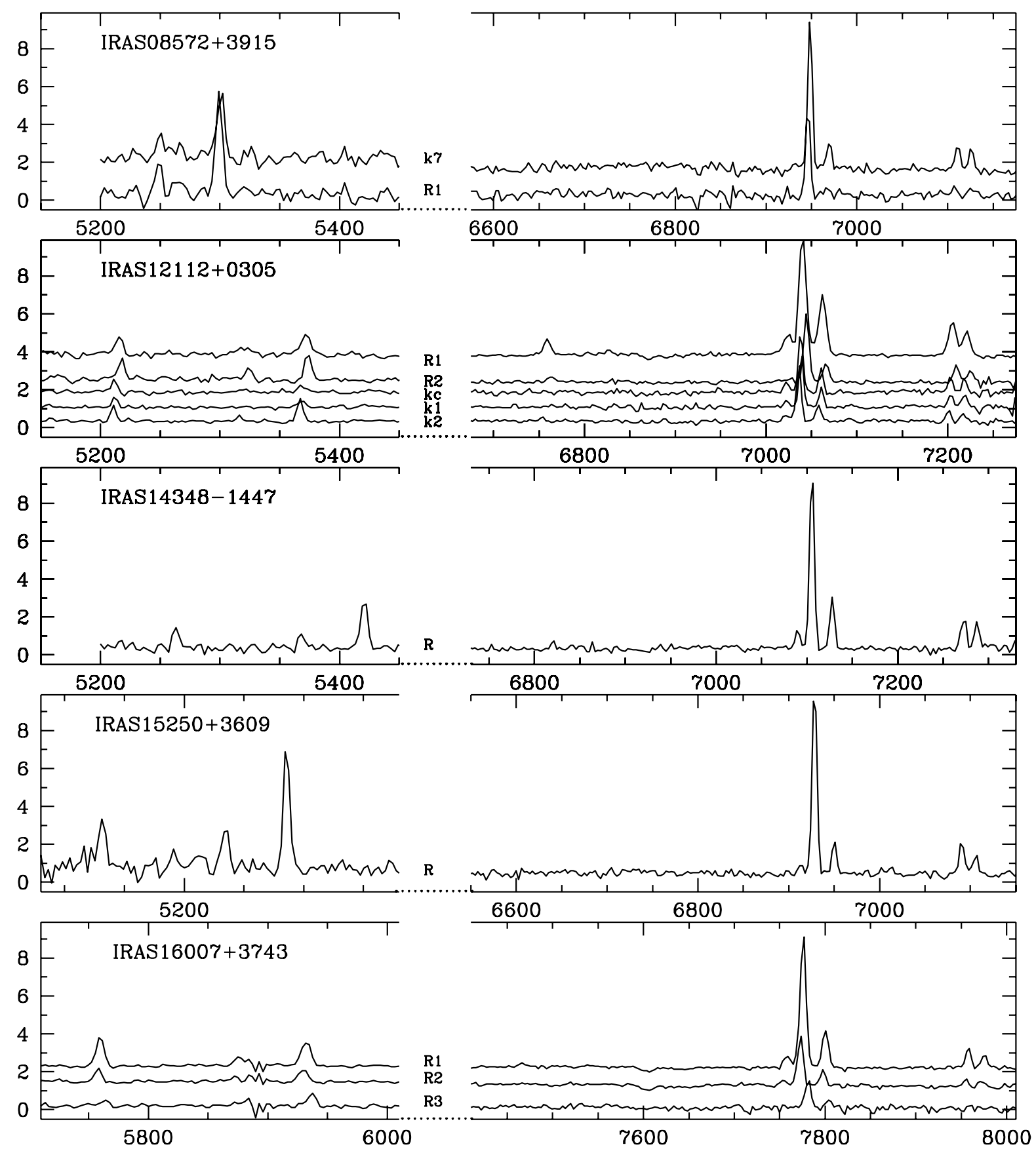

Wavelength $(\AA)$

Fig. 2. Representative observed spectra of the main extranuclear line-emitting regions. Note that the horizontal axis does not represent a continuous range in wavelength, but two subsets of the full INTEGRAL spectrum corresponding to $\mathrm{H} \beta-[\mathrm{O}$ III] $\lambda \lambda 4959,5007$ and [O I] $\lambda 6300-$ $\mathrm{H} \alpha+[\mathrm{N}$ II $] \lambda \lambda 6548,6586-[\mathrm{S} \mathrm{II}] \lambda \lambda 6717,6731$ spectral regions, respectively. Flux units are arbitrary and for the sake of clarity, offsets have been applied to the spectra.

IRAS 12112+0305: This system shows several regions of interest. The brightest emission line region (R1) is not associated with any of the nuclei of the system, but with a low surface brightness region in the WFPC2 image towards the east of the system (Colina et al. 2000). This image also shows an arc-like condensation located $3^{\prime \prime}$ north of the southern nucleus at $\mathrm{PA} \sim 20^{\circ}(\mathrm{R} 2)$ which is relatively bright in the $\mathrm{H} \alpha$ emission line map. Finally, the southern tidal tail shows a series of condensations which correspond either to peaks in the emission line map (k1 and $\mathrm{k} 2$ ) or in the continuum map (kc).
IRAS 14348-1447: The most interesting region in this system was identified by Mihos \& Bothun (1998) as a peak of $\mathrm{H} \alpha$ emission towards the southeast of the system (R). In addition, two other regions should be mentioned here. On the one hand, the peak of $\mathrm{H} \alpha$ emission in the northern galaxy is not associated with its nucleus but with four knots located at the base of its tidal tail. Similarly to IRAS $15206+3342$, the individual knots cannot be resolved in the ground-based data and its analysis is not considered here. On the other hand, the WFPC2 image shows a faint condensation at $\sim 4$.' 0 and 
$\mathrm{PA} \sim 70^{\circ}$ from the southern nucleus whose analysis is also not considered here due to the elevated flux contamination from the southern galaxy in the INTEGRAL data.

IRAS 15250+3609: This galaxy presents a low surface brightness region in the $F 814 \mathrm{~W}$ band at $\sim 77^{\prime \prime} 0$ from the nucleus towards the southwest (R), which is however relatively bright in the line emission maps (Monreal-Ibero 2004). HST infrared images show a series of knots in the main body of the galaxy probably associated with globular clusters (Scoville et al. 2000). Although they can be responsible for some of the extended emission observed in the ground-based data, they cannot be identified as individual sources and their analysis is not considered here.

IRAS 16007+3743: The morphology of IRAS 16007+3743 could indicate that this system is a multiple merger (Cui et al. 2001; Borne et al. 2000). However, kinematical information derived from INTEGRAL (i.e. velocity field and velocity dispersion maps) led us to classify this system as a system of two spiral galaxies in interaction (García-Marín 2005). Three regions have been identified: $\mathrm{R} 1$ and $\mathrm{R} 2$ seen in the $\mathrm{H} \alpha$ map as two bright condensation along the northern tidal tail and R3, a region at $\sim 3$.' $^{\prime} 0$ towards the south of the system apparently not associated with any of the tidal tails.

To have an idea of the data quality, the observed spectra for the regions under study are shown in Fig. 2. From blue to red, $\mathrm{H} \beta,[\mathrm{O}$ III $] \lambda \lambda 4959,5007,[\mathrm{O} \mathrm{I}] \lambda 6300, \mathrm{H} \alpha+[\mathrm{N} \mathrm{II}] \lambda \lambda 6548,6584$ and $[\mathrm{S}$ II $] \lambda \lambda 6717,6731$ are detected in most of the regions with enough $\mathrm{S} / \mathrm{N}$ ratio.

\subsection{Characterisation of the external star-forming regions}

\subsubsection{Line ratios}

Measured line ratios are shown in Table 2 and Fig. 3, which contain the classical diagnostic diagrams of Veilleux \& Osterbrock (1987). The regions present line ratios typical of H II regions. It is only by means of the $[\mathrm{OI}] \lambda 6300 / \mathrm{H} \alpha$ line ratio that some of them could marginally be classified as LINER. Star formation is the most plausible mechanism to explain the observed line ratios i.e. the line ratio of all the regions analysed here are well within the limits proposed by Kewley et al. (2001) for ionisation by star formation.

In general, line ratios are comparable to those found in TDG candidates (e.g. Duc \& Mirabel 1998; Temporin et al. 2003; López-Sánchez et al. 2004), except for their [O I] $\lambda 6300 / \mathrm{H} \alpha$ which is smaller by $\sim 0.3 \mathrm{dex}$ in those cases where it could be measured. However, the poor statistics (i.e. the [O I] 66300 line could only be measured for four regions) makes it difficult to assess the significance of this result.

\subsubsection{Metallicities}

One observational characteristic that helps to establish the tidal origin of a certain dwarf galaxy is its high metallicity, which suggests that TDGs are made up from processed material (Duc $\&$ Mirabel 1998). Abundances are usually estimated using empirical methods based on the intensities of certain optical lines such as the widely used method of the $R_{23}$ calibrator (i.e. TorresPeimbert et al. 1989) and the $S_{23}$ indicator (Vílchez \& Esteban 1996). However, both involve emission lines which are not available within our spectral range. Nevertheless, it is possible to estimate the metallicity using the N2 calibrator proposed by Denicoló et al. (2002) - based on the ratio between the
[N II] $\lambda 6584$ and $\mathrm{H} \alpha$ emission lines -, and the empirical diagrams of Edmunds \& Pagel (1984), - based on the [O III] $\lambda \lambda 4959,5007$ to $\mathrm{H} \beta$ ratio - parametrised as explained in Duc \& Mirabel (1998). The predicted metallicity values expected from these indicators are shown in the last two columns of Table 2. Both indicators predict similar values for the metallicity (average of $12+\log \mathrm{O} / \mathrm{H}=8.70$ for the $\mathrm{N} 2$ calibrator against 8.57 for that involving the oxygen lines). Given that typical uncertainties for the line ratios are about 0.2 dex (which translates into $\sim 0.3$ for the metallicity), differences between both indicators are not significative. These relatively high metallicities are about 2 times larger than those derived for other TDGs candidates ( $\sim 8.35$, Duc $\&$ Mirabel 1998; Weilbacher et al. 2003) and about half the solar metallicity.

\subsubsection{Sizes}

Sizes were estimated from the WFPC2/F814W images. The irregular shape of the regions as well as some possible contamination from other structures within the system (i.e. other regions, tails or the parent galaxy itself) made it difficult to define their limits. In order to proceed in a systematic way and compare with the sizes of other extragalactic objects, we defined the size of a given region as the area encircled in the largest closed contour centred in the emission peak associated with that region. This allows us to define an equivalent radius as $r_{\text {equ }}=\sqrt{\text { Area } / \pi}$ which gives an estimate of the total size of the region. An effective radius was also measured as that containing half of the flux within this area (see Col. 7 and 8 of Table 3). They can be used for comparison with the characteristic radii derived for other objects. The ratio between the effective and equivalent radii $(0.3-0.7)$ gives and idea of the compactness of a certain condensation.

Equivalent radii range from a few hundreds pcs to $\sim 1.5 \mathrm{kpc}$ (mean of $750 \mathrm{pc}$ ). In general, when comparing with the sizes of H II regions (which typically have radii of $\sim 100-900 \mathrm{pc}$ ), the present regions are similar to the largest Giant H II regions (Kennicutt 1984; Mayya 1994).

All the regions under study have effective radii (mean $\sim 430$ pc) comparable to dwarf galaxies located in the Local Group ( $r \sim 0.3 \mathrm{kpc}$, Mateo 1998), to the so-called Blue Compact Dwarf galaxies, with effective radii $0.2 \lesssim r_{\text {eff }} \lesssim 1.8 \mathrm{kpc}$ (e.g. Marlowe et al. 1997; Cairós et al. 2003) or to some already detected TDG (e.g. Duc \& Mirabel 1998).

\subsection{4. $\mathrm{H} \alpha$ equivalent widths and luminosities}

$\mathrm{H} \alpha$ equivalent width and luminosity distributions are shown in Fig. 4 together with the values measured for some TDGs as well as H II regions taken from the literature. The relatively high $\mathrm{H} \alpha$ equivalent widths derived (i.e. 34-257 $\AA$ ), indicative of the existence of a very young stellar population, are comparable to those measured for TDG candidates (e.g. 12-623 for Iglesias-Páramo \& Vílchez 2001 or 24-198 Å for Temporin et al. 2003) and for extragalactic H II regions in general (Mayya 1994). Regarding the $\mathrm{H} \alpha$ luminosities, once the extinction effects have been taken into account, the selected regions are more luminous than the Giant H II Regions (Mayya 1994; Kennicutt 1984). In general, these luminosities are in better agreement with those found for TDG candidates (Duc \& Mirabel 1998; Iglesias-Páramo \& Vílchez 2001). The most suitable sample for comparison with our candidates is that of Temporin et al. (2003) which takes into account extinction effects. With the exception of three very bright regions, the $\mathrm{H} \alpha$ luminosities are comparable 
Table 2. Properties of the star-forming regions (I): emission line ratios.

\begin{tabular}{|c|c|c|c|c|c|c|c|c|c|}
\hline Region & $\begin{array}{c}\Delta \alpha^{a} \\
\left({ }^{\prime \prime}\right)\end{array}$ & $\begin{array}{r}\Delta \delta^{a} \\
\left({ }^{\prime \prime}\right)\end{array}$ & $E(B-V)^{b}$ & {$[\mathrm{O} \mathrm{III}] / \mathrm{H} \beta^{b, c}$} & {$[\mathrm{O} \mathrm{I}] / \mathrm{H} \alpha^{b, c}$} & {$[\mathrm{~N}$ II $] / H \alpha^{b, c}$} & {$[\mathrm{~S}$ II $] / \mathrm{H} \alpha^{b, c}$} & $\begin{array}{l}12+\log (\mathrm{O} / \mathrm{H})^{d} \\
\left([\mathrm{O} \text { III }] / \mathrm{H} \beta_{\text {upper }}\right)\end{array}$ & $\begin{array}{c}12+\underset{\log (\mathrm{O} / \mathrm{H})^{e}}{(\mathrm{~N} 2)} \\
\end{array}$ \\
\hline \multicolumn{10}{|c|}{ IRAS $08572+3915^{f}$} \\
\hline $\mathrm{R} 1^{g}$ & 5.2 & -7.4 & $\ldots$ & 0.24 & $\ldots$ & -0.85 & $\ldots$ & 8.49 & 8.50 \\
\hline k7 & 5.3 & 2.2 & $\ldots$ & 0.54 & $\ldots$ & -0.82 & $\ldots$ & 8.29 & 8.52 \\
\hline \multicolumn{10}{|c|}{ IRAS $12112+0305$} \\
\hline R1 & 2.7 & -1.5 & 0.25 & 0.22 & -1.24 & -0.66 & -0.58 & 8.50 & 8.64 \\
\hline $\mathrm{R} 2$ & -1.5 & 0.6 & 1.07 & 0.01 & -1.02 & -0.41 & -0.43 & 8.65 & 8.82 \\
\hline $\mathrm{kc}$ & -1.8 & -7.4 & 0.47 & 0.16 & $\ldots$ & -0.64 & -0.99 & 8.55 & 8.65 \\
\hline $\mathrm{k} 1$ & -2.8 & -7.2 & 0.66 & -0.12 & $\ldots$ & -0.49 & -0.38 & 8.74 & 8.76 \\
\hline $\mathrm{k} 2$ & 0.7 & -7.7 & 0.45 & 0.08 & $\ldots$ & -0.42 & -0.39 & 8.60 & 8.81 \\
\hline \multicolumn{10}{|c|}{ IRAS $14348-1447$} \\
\hline $\mathrm{R}$ & 5.1 & -1.7 & 1.12 & 0.26 & -1.36 & -0.55 & -0.49 & 8.48 & 8.72 \\
\hline \multicolumn{10}{|c|}{ IRAS $15250+3609$} \\
\hline $\mathrm{R}$ & -5.0 & -4.8 & 0.25 & 0.26 & $\ldots$ & -0.78 & -0.60 & 8.48 & 8.55 \\
\hline \multicolumn{10}{|c|}{ IRAS $16007+3743$} \\
\hline R1 & 0.9 & 5.4 & 0.55 & 0.13 & -1.05 & -0.47 & -0.67 & 8.59 & 8.78 \\
\hline R2 & 0.7 & 2.8 & 0.40 & -0.02 & $\ldots$ & -0.55 & -0.67 & 8.65 & 8.71 \\
\hline R3 & 2.2 & -1.9 & 0.75 & 0.49 & $\ldots$ & -0.55 & $\ldots$ & 8.59 & 8.75 \\
\hline
\end{tabular}

${ }^{a}$ Relative positions of the regions. We took the northern nucleus as the reference in IRAS $08572+3915$ and IRAS 12112+0305; the southern one in IRAS 14348-1447 and the western one in IRAS 16007+3743.

${ }^{b}$ Calculated as the average value in a 0 '. 45 -radius aperture centred in the region of interest.

$c$ Extinction corrected data. We assumed a foreground screen model, and used the reddening curve of Whitford (1958) parametrised as explained in Miller \& Mathews (1972) and $R=3.1$ (Rieke \& Lebofsky 1985), except for regions R1 and k7 of IRAS 08572+3915 where no extinction measurements are available. Typical errors for line ratios are $\lesssim 0.2$ dex.

$d$ Derived metallicities using the upper branch of the empirical diagram of Edmunds \& Pagel (1984).

$e$ Derived metallicities using the N2 calibrator of Denicoló et al. (2002).

$f$ Measured values taken from Arribas et al. (2000).

$g$ This corresponds to knots k4 and k5 in the notation of Arribas et al. (2000). Their relative positions are: (5.8, -7.8) and (4.7, -7.0).

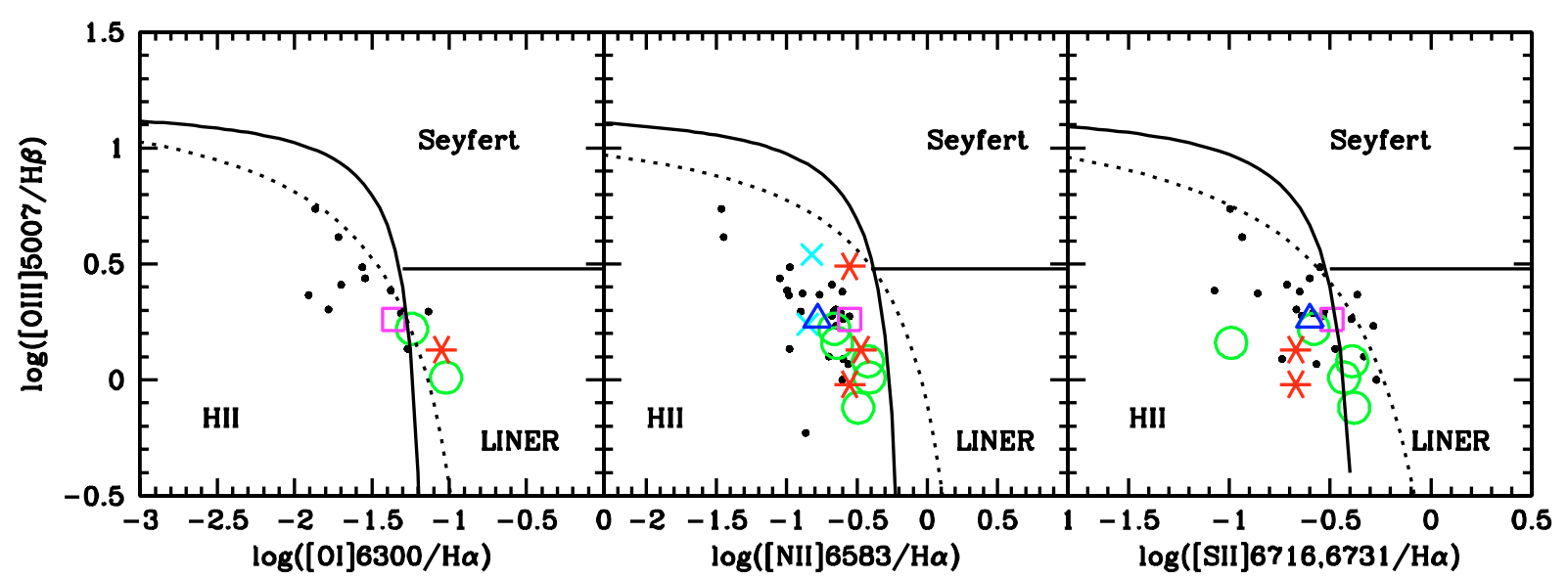

Fig. 3. Position of the different regions in the diagnostic diagrams proposed by Veilleux \& Osterbrock (1987). The solid lines show the empirical borders found by these authors between the different ionisation types while the dashed lines show the theoretical borders proposed by Kewley et al. (2001) to delimit the area where the line ratios can be explained by star formation. The colour/symbol code used is: IRAS 08572+3915, cyan/cross; IRAS 12112+0305, green/circle; IRAS 14348-1447, magenta/square; IRAS 15250+3609, blue/triangle; IRAS 16007+3743, red/asterisk. Typical errors are $\sim 0.2$ dex. The measured ratios in other TDG candidates are shown for comparison as black dots (Duc \& Mirabel 1998; Temporin et al. 2003; López-Sánchez et al. 2004). (This figure is available in color in electronic form.)

to those of Temporin et al. (mean $\sim 3 \times 10^{40} \mathrm{erg} \mathrm{s}^{-1}$ for their sample against $\sim 6 \times 10^{40} \mathrm{erg} \mathrm{s}^{-1}$ for ours).

The extinction corrected luminosity of three regions are well above $5 \times 10^{41} \mathrm{erg} \mathrm{s}^{-1}$ which is more than one order of magnitude larger than the luminosities found in typical TDGs. Two of them belong to IRAS $16007+3743$ which is at $z=0.185$ and has a factor of 2-3 lower linear resolution than the rest of the sample. One possibility is that emission from these two particular regions was due to a wider area that encompasses more than one condensation. However, a resolution effect cannot be used as explanation for the high luminosity of the third region ( $\mathrm{R} 2$ of IRAS $12112+0305$, scale $\sim 1.4 \mathrm{kpc} \operatorname{arcsec}^{-1}$ ). Alternatively, it may be possible that the relatively large amount of gas involved in ULIRGs in general, and the violent event suggested by the morphology of IRAS $16007+3743$ and IRAS $12112+0305$ could lead to the large luminous TDGs.

It is widely known that $\mathrm{H} \alpha$ emission can be used to constrain the properties of recent episodes of star formation. An upper limit to the age can be estimated by using its equivalent width, as it decreases with time (see inset in Fig. 5). We 
Table 3. Properties of the star-forming regions (II): $\mathrm{H} \alpha$ related observables, I magnitudes, sizes and distances.

\begin{tabular}{|c|c|c|c|c|c|c|c|c|c|}
\hline Region & $\begin{array}{c}E W(\mathrm{H} \alpha)^{a} \\
(\AA)\end{array}$ & $\begin{array}{c}F_{\text {obs }}(\mathrm{H} \alpha)^{b} \\
\left(10^{-16} \mathrm{erg} \mathrm{s}^{-1} \mathrm{~cm}^{-2}\right)\end{array}$ & $\begin{array}{c}L(\mathrm{H} \alpha)^{c} \\
\left(10^{40} \mathrm{erg} \mathrm{s}^{-1}\right)\end{array}$ & $m_{I}^{d}$ & $M_{I}^{e}$ & $\begin{array}{l}r_{\text {equ }} \\
(\mathrm{pc})\end{array}$ & $\begin{array}{l}r_{\text {eff }} \\
(\mathrm{pc})\end{array}$ & $\begin{array}{l}D_{\mathrm{CM}}^{f} \\
(\mathrm{kpc})\end{array}$ & $\begin{array}{l}D_{\text {near }}^{g} \\
(\mathrm{kpc})\end{array}$ \\
\hline \multicolumn{10}{|c|}{ IRAS $08572+3915^{\mathrm{h}}$} \\
\hline $\mathrm{R} 1$ & 78 & 7.0 & 0.6 & 23.78 & -13.30 & 373 & 121 & 8.8 & 4.2 \\
\hline $\mathrm{k} 7$ & 245 & 4.0 & 0.3 & 22.62 & -14.46 & 239 & 128 & 6.4 & 6.4 \\
\hline \multicolumn{10}{|c|}{ IRAS $12112+0305$} \\
\hline $\mathrm{R} 1$ & 257 & 31.8 & 7.4 & 21.12 & -16.86 & 373 & 224 & 5.0 & 4.3 \\
\hline $\mathrm{R} 2$ & 79 & 37.4 & 57.6 & 19.05 & -20.14 & 698 & 494 & 2.4 & 2.2 \\
\hline $\mathrm{kc}$ & 34 & 2.7 & 1.0 & 21.15 & -17.16 & 451 & 311 & 9.0 & 6.9 \\
\hline $\mathrm{k} 1$ & 46 & 3.5 & 2.1 & 21.43 & -17.16 & 383 & 150 & 9.0 & 7.1 \\
\hline $\mathrm{k} 2$ & 71 & 3.4 & 1.2 & 22.02 & -16.26 & 378 & 249 & 9.0 & 8.4 \\
\hline \multicolumn{10}{|c|}{ IRAS 14348-1447 } \\
\hline $\mathrm{R}$ & 194 & 15.5 & 34.1 & 20.40 & -19.13 & 1082 & 546 & 8.2 & 6.0 \\
\hline \multicolumn{10}{|c|}{ IRAS $15250+3609$} \\
\hline $\mathrm{R}$ & 141 & 6.5 & 0.6 & 20.73 & -16.36 & 891 & 420 & 6.9 & 6.9 \\
\hline \multicolumn{10}{|c|}{ IRAS $16007+3743$} \\
\hline $\mathrm{R} 1$ & 40 & 12.0 & 57.1 & 20.16 & -20.63 & 1151 & 828 & 17.1 & 16.9 \\
\hline $\mathrm{R} 2$ & 234 & 35.5 & 189.5 & 19.80 & -21.07 & 1375 & 884 & 9.4 & 8.9 \\
\hline R3 & 80 & 5.85 & 8.8 & 21.83 & -19.23 & 1562 & 851 & 5.8 & 9.0 \\
\hline
\end{tabular}

${ }^{a}$ Calculated as the average value in an 0.'45-radius aperture centred in the region of interest.

$b$ As a compromise between collecting all the flux of the region and avoiding the contamination from the neighbouring zones of the systems, flux was measured in a 1'.0-radius aperture in all regions of IRAS 08572+3915, IRAS 14348-1447, IRAS $15250+3609$ and R1 and R2 of IRAS $12112+0305$ while for those in the tidal tail of this system and those in IRAS 16007+3743, a 0' 5 -radius aperture was used.

${ }^{c}$ Extinction corrected $\mathrm{H} \alpha$ luminosity except for regions R1 and k7 of IRAS $08572+3915$ where no extinction measurements are available.

${ }^{d}$ Observed $I$ magnitude, measured within the area enclosed by the biggest closed contour centred in the emission peak (see explanation in text).

$e$ Extinction corrected absolute $I$ magnitude.

$f$ Distance to the mass centre of the system.

$g$ Distance to the nearest galaxy.

${ }^{h} \mathrm{H} \alpha$ equivalent widths and fluxes taken from Arribas et al. (2000).

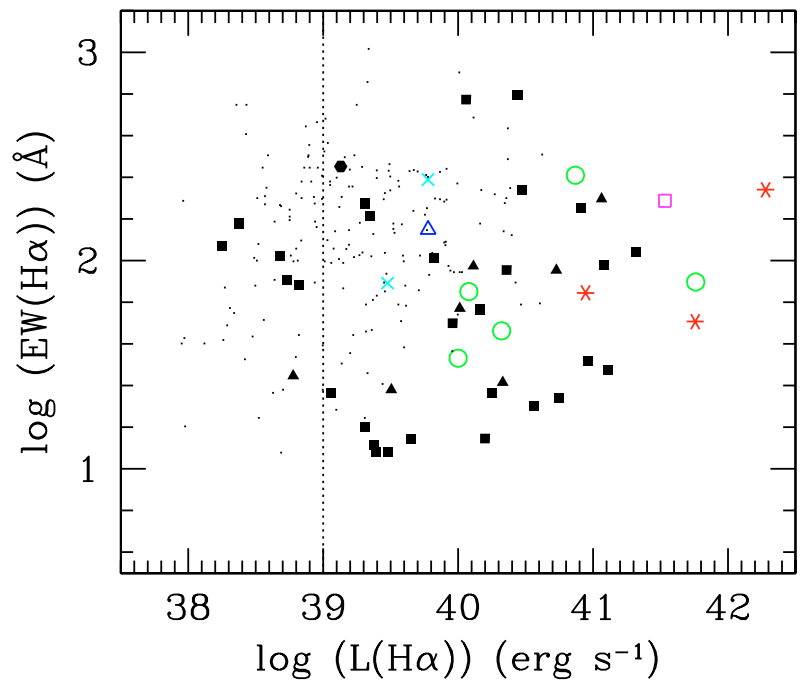

Fig. 4. $\mathrm{H} \alpha$ luminosities vs. equivalent widths. Data from the present study follow the same colour/symbol code as in Fig. 3. Comparison measurements for the extragalactic H II regions of Mayya (1994) (dots, not extinction corrected) and for other extranuclear star forming regions which have been analysed as possible TDG candidates (solid symbols) are shown. Those of Iglesias-Páramo \& Vílchez (2001) (squares) and Mundell et al. (2004) (hexagon) are not extinction corrected while those of Temporin et al. (2003) (triangles) are. The vertical dashed line shows the luminosity limit for TDG candidates used in Iglesias-Páramo \& Vílchez (2001). (This figure is available in color in electronic form.)

used STARBURST99 models (Leitherer et al. 1999) to estimate ages for the bursts in the extranuclear regions. In view of the estimated metallicities in Sect. 4.2.2, we chose two different

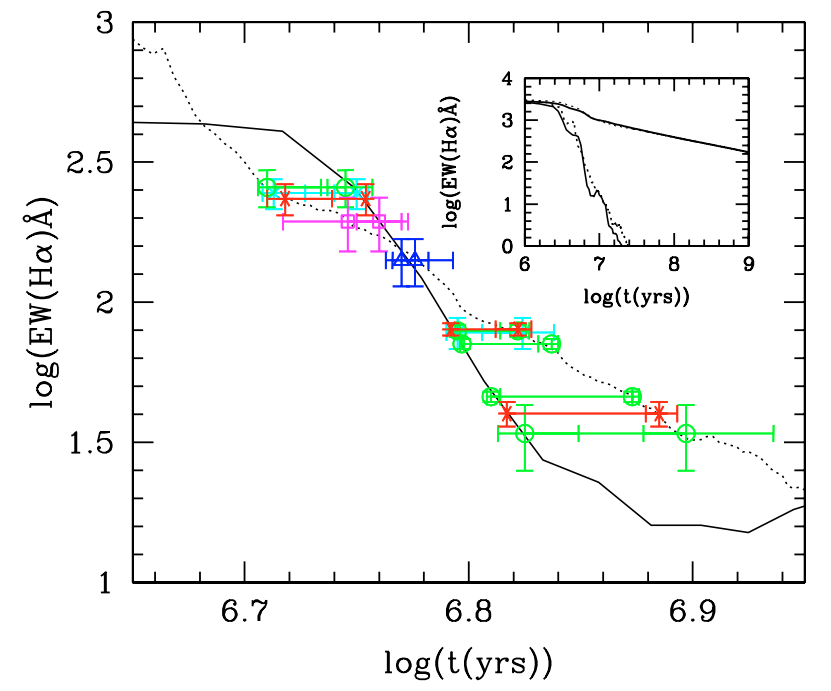

Fig. 5. Measured $\mathrm{H} \alpha$ equivalent widths in comparison to two SB99 instantaneous bursts of $Z=0.020$ (continuum line) and $Z=0.008$ (dashed line). Colour/symbol code is the same as in Fig. 3. The small inset shows the covered range in equivalent width for both continuous and instantaneous STARBURSTS99 models (thin and thick lines respectively). (This figure is available in color in electronic form.)

spectral synthesis models, both for an instantaneous burst with a Salpeter IMF, and upper mass limit of $125 M_{\odot}$ but with two different metallicities: $Z=0.020$ and $Z=0.008$. Models with continuous star formation cannot explain the observed equivalent widths as the newly born massive stars are able to maintain values of the equivalent width which are too elevated in comparison to those observed even for ages greater than 100 Myrs. The 


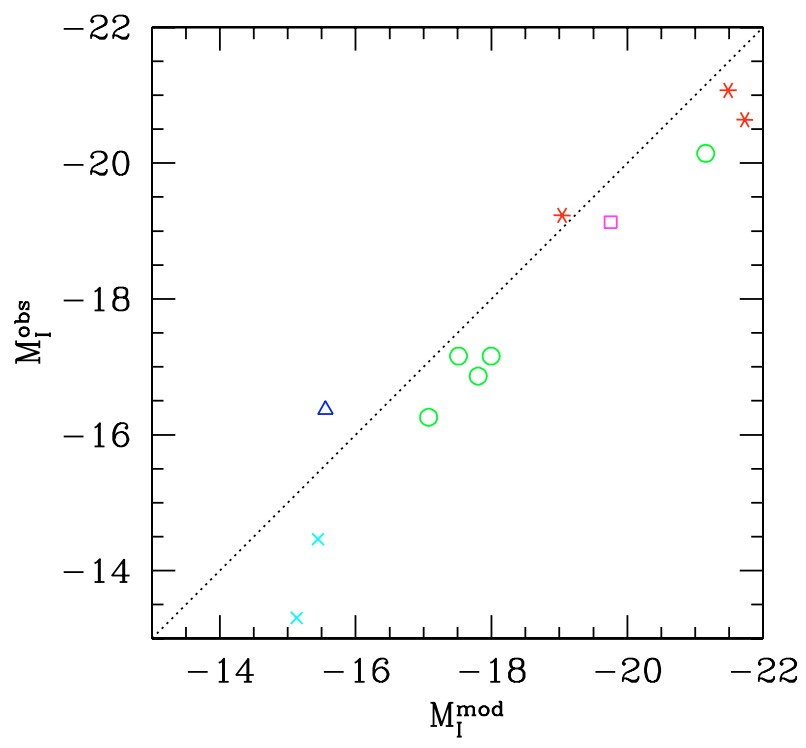

Fig. 6. Comparison between measured and predicted $I$ magnitudes. Colour/symbol code is the same as in Fig. 3. The dotted line represents slope one. Predicted magnitudes were obtained as an average of the predicted magnitudes for the $Z=0.020$ and $Z=0.008$ instantaneous burst models. (This figure is available in color in electronic form.).

estimated ages for the bursts are shown in Fig. 5 and second column of Table 4. They range from 5.5 to $7.1 \mathrm{Myr}$ if the model with solar metallicity is assumed or from 5.1 to $7.8 \mathrm{Myr}$ if that for lower metallicity is used, confirming the presence of a young population.

Summarising, equivalent widths of the analysed regions are similar to those derived for both extragalactic $\mathrm{H}$ II regions and TDG candidates and are typical of young burst of star formation. The $\mathrm{H} \alpha$ luminosity place these regions more in the group of TDG candidates than in that of extragalactic $\mathrm{H}$ II regions.

\subsubsection{I magnitudes}

We have measured the I magnitudes within the apertures defined in Sect. 4.2.3 using the task POLYPHOT under the IRAF environment. Typical errors in the measurements where $\sim 0.1$, with the exception of R1 in IRAS $08572+3915$ which was $\sim 0.4$. The comparison between these magnitudes and those derived from STARBURST99 are shown in Fig. 6 where plotted magnitudes were obtained as an average of the predicted magnitudes for the $Z=0.020$ and $Z=0.008$ instantaneous burst models. The trend is good although the predicted values from extinction corrected $\mathrm{H} \alpha$ luminosities overestimate the observed magnitudes by $\sim 0.9$ mag on average. These differences can be explained by both the uncertainties associated with the selection of the aperture in the different bands and the existence of other stellar populations either from a previous burst during the merging process or from the original population in the merging galaxies, as found in some TDG candidates (e.g. López-Sánchez et al. 2004). The presence of such an older population would cause an underestimation of the equivalent width and thus, overestimation of the age of the burst. Younger ages for the bursts translate into a decrease of the predicted $I$ magnitudes of $\sim 0.5-1.0$ mag according to the models.

\subsubsection{Distances}

The two last columns in Table 3 show the projected distances between the studied regions and the mass centre of the system, as well as the closer galaxy. Typical measured distances are $\sim 8 \mathrm{kpc}$ with a maximum of $\sim 17 \mathrm{kpc}$. However, these objects were not selected based on a particular favourable geometry. Indeed, their geometries are very complex, and the influence of projection effects - which should be present - is particularly difficult to quantify. These distances have to be seen as lower limits to the current ones. These are relatively close distances to the parent galaxy when compared with other condensations in $\mathrm{H} \alpha$ identified as TDGs (e.g. Duc \& Mirabel 1998) although there are already some examples of TDG candidates at such distances (e.g. TD44a in Iglesias-Páramo \& Vílchez 2001 or objects A2 and E in Amram et al. 2004).

\section{Discussion: what are the chances of survival of the analysed regions as TDGs?}

Most of the observational properties of the selected regions (i.e. $\mathrm{H} \alpha$ equivalent widths and luminosities, metallicities, ionisation states and radii) derived in Sect. 4 place these regions in the locus of the most luminous extragalactic Giant H II Regions and are consistent with those expected for TDGs or TDG progenitors. In the next section, we use the kinematical information provided by INTEGRAL which, together with the evolutive state of the system, allows us to estimate the likelihood of survival of these candidates.

As TDGs are stable entities with their own dynamic, the best definition for this kind of object is that proposed by Duc et al. (2000) who identify a TDG as the object that constitutes a selfgravitating entity and that was made up from the debris of a galaxy interaction. To evaluate the chance of survival of TDG candidates, two basic questions need to be answered: i) Is the candidate massive enough to survive its internal movements? ii) Is the candidate massive enough to survive to gravitational forces exerted by the parent galaxy? Depending on the available observables, in the past several criteria have been used in the literature to classify a certain condensation as a TDG. In the following we apply them (when possible) to our star-forming regions.

\subsection{Stability against internal motions}

Iglesias-Páramo \& Vílchez (2001) established a luminosity criterion $\left(L(\mathrm{H} \alpha)>10^{39} \mathrm{erg} \mathrm{s}^{-1}\right)$ which should fulfil systems stable to internal motions. As mentioned above (Sect. 4.2.4), all selected candidates meet this criterion (see also Fig. 4), especially two very luminous regions of IRAS $16007+3743$ and R2 in IRAS $12112+0305$.

One of the best tests to check if a certain detected condensation constitutes a self-gravitating entity is to detect velocity gradients that could indicate the existence of independent rotation (e.g. Weilbacher et al. 2002; Bournaud et al. 2004; Mendes de Oliveira et al. 2001). However our data lack the necessary angular resolution to resolve any velocity field across the extranuclear condensations. Typical size of the detected regions is roughly equivalent to 1-3 SB2 fibres, which does not allow derivation the individual velocity field of the regions under study.

Another method that can be used to establish whether or not the TDG candidates are stable, self-gravitating entities is to study their location in the radius-velocity dispersion correlation measured for ellipticals and globular clusters. To this end, we plotted the classical fits in the $r_{\mathrm{eq}}-\sigma$ plane for elliptical galaxies, 
Table 4. Properties of the star-forming regions (III): derived characteristics of the stellar populations and dynamical parameters.

\begin{tabular}{|c|c|c|c|c|c|c|c|c|c|}
\hline Region & $\begin{array}{c}\mathrm{Age}^{a} \\
\left(10^{6} \mathrm{Myr}\right)\end{array}$ & $\begin{array}{c}m_{y . b .}^{a} \\
\left(10^{6} M_{\odot}\right)\end{array}$ & $\begin{array}{c}\sigma^{b} \\
\left(\mathrm{~km} \mathrm{~s}^{-1}\right)\end{array}$ & $\begin{array}{c}M_{\mathrm{dyn}} \\
\left(10^{8} M_{\odot}\right)\end{array}$ & $\begin{array}{c}M_{\text {tid }}^{\mathrm{A} c} \\
\left(10^{8} M_{\odot}\right)\end{array}$ & $\begin{array}{c}M_{\mathrm{tid}}^{\mathrm{B} d} \\
\left(10^{8} M_{\odot}\right)\end{array}$ & $\begin{array}{c}v_{\text {rel }} \\
\left(\mathrm{km} \mathrm{s}^{-1}\right)\end{array}$ & $\begin{array}{c}v_{\mathrm{esc}} \\
\left(\mathrm{km} \mathrm{s}^{-1}\right)\end{array}$ & $M_{\mathrm{dyn}} / M_{\mathrm{tid}}$ \\
\hline \multicolumn{10}{|c|}{ IRAS $08572+3915$} \\
\hline R1 & 5.4 & 1.9 & & $\ldots$ & 0.04 & 0.09 & 196 & 133 & $\ldots$ \\
\hline $\mathrm{k} 7$ & 6.4 & 2.2 & 47 & 5.9 & 0.03 & 0.02 & 41 & 156 & 196 \\
\hline \multicolumn{10}{|c|}{ IRAS $12112+0305$} \\
\hline R1 & 5.3 & 22.8 & 60 & 16.8 & 1.22 & 1.10 & 75 & 410 & 14 \\
\hline $\mathrm{R} 2$ & 6.4 & 420.6 & 56 & 32.4 & 72.32 & 53.65 & -280 & 592 & 0.4 \\
\hline $\mathrm{kc}$ & 7.3 & 11.5 & $\ldots$ & $\ldots$ & 0.37 & 0.32 & -338 & 306 & $\ldots$ \\
\hline k1 & 6.9 & 19.6 & $\ldots$ & $\ldots$ & 0.23 & 0.21 & -207 & 306 & $\ldots$ \\
\hline $\mathrm{k} 2$ & 6.6 & 9.4 & $\ldots$ & $\ldots$ & 0.18 & 0.11 & -343 & 306 & $\ldots$ \\
\hline \multicolumn{10}{|c|}{ IRAS 14348-1447 } \\
\hline $\mathrm{R}$ & 5.7 & 136.6 & 50 & 28.5 & 10.6 & 14.78 & 144 & 402 & 1.9 \\
\hline \multicolumn{10}{|c|}{ IRAS $15250+3609$} \\
\hline $\mathrm{R}$ & 6.5 & 2.8 & 50 & 21.9 & 3.62 & 3.62 & 170 & 264 & 6.0 \\
\hline \multicolumn{10}{|c|}{ IRAS $16007+3743$} \\
\hline R1 & 7.1 & 581.6 & 61 & 64.4 & 1.7 & 1.1 & -41 & 302 & 38 \\
\hline $\mathrm{R} 2$ & 5.4 & 678.3 & 76 & 106.8 & 17.7 & 112.39 & 92 & 408 & 0.9 \\
\hline $\mathrm{R} 3$ & 6.4 & 62.8 & 85 & 128.5 & 106.65 & 12.58 & 338 & 519 & 1.2 \\
\hline
\end{tabular}

a The age and mass in young stars obtained as the average from the predictions for the low and high metallicity models.

${ }^{b}$ Calculated as the average value in an 0.'45-radius aperture centred in the region of interest.

$c$ Tidal mass assuming the potential is created by a point mass in the mass centre of the system.

$d$ Tidal mass assuming the potential is created by the nearest galaxy.

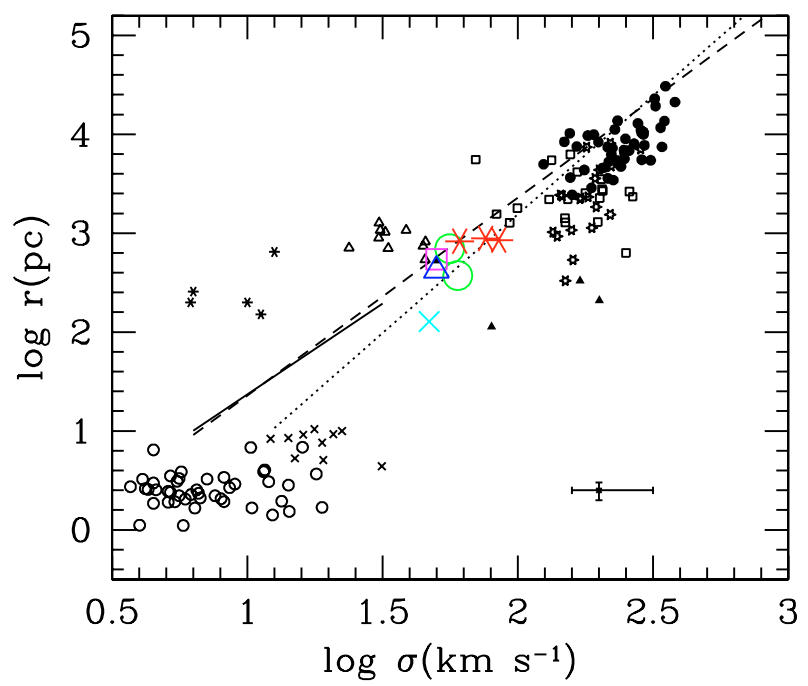

Fig. 7. Velocity dispersion vs. estimated effective radius. The colour/symbol code is the same as in Fig. 3. The typical size for errors is shown in the lower right corner. The continuous line represents the fit for extragalactic H II regions obtained by Terlevich \& Melnick (1981) while the dashed and dotted lines show their fit for elliptical galaxies, and globular cluster + elliptical galaxies respectively. Small symbols represent different samples of dynamically hot systems: open circles, galactic globular clusters (Trager et al. 1993; Pryor \& Meylan 1993); crosses, massive globular clusters in NGC 5128 (Martini \& Ho 2004); open triangles, dwarf elliptical galaxies (Geha et al. 2003); open squares, intermediate ellipticals; solid circles, giant ellipticals; solid triangles, compact ellipticals; asterisks, dwarf ellipticals; and stars, bulges (all from Bender et al. 1992). (This figure is available in color in electronic form.)

globular clusters and H II regions of Terlevich \& Melnick (1981) together with our data in Fig. 7. For the present INTEGRAL configuration the spectral resolution is $\sigma \sim 80 \mathrm{~km} \mathrm{~s}^{-1}$. We considered that we were able to determine a reliable measurement of the velocity dispersion when this was greater than half the spectral resolution. We also overplotted the position of recent samples of hot dynamically systems such as galactic globular clusters (Trager et al. 1993; Pryor \& Meylan 1993), massive globular clusters in in NGC5128 (Martini \& Ho 2004), dwarf elliptical galaxies in the Virgo cluster (Geha et al. 2003), and the sample of dynamically hot systems of Bender et al. (1992). Based on their position in the radius-velocity dispersion diagram, the selected TDG candidates should be self-gravitating entities. Moreover, they are in a region close to other dwarf galaxies (compare in particular with the sample of Geha et al. 2003), and far from the region occupied by globular clusters.

\subsection{Stability against forces from the parent galaxy}

One way to see whether a condensation is stable against the gravitational potential of the parent galaxy/system is by estimating its tidal mass (Binney \& Tremaine 1987; Mendes de Oliveira et al. 2001) which is defined as:

$M_{\mathrm{tid}}=3 M\left(\frac{R}{D}\right)^{3}$

where $M$ is the mass of the parent galaxy, $R$ is the radius of the TDG candidate (here estimated as $r_{\text {equ }}$ ) and $D$ is the distance to the parent galaxy. If the tidal mass is smaller than the mass for the TDG candidate, then the candidate is stable against the forces exercised by the parent galaxy. This expression is valid in those cases where the size of a certain region is small in comparison with the distance to the parent galaxy (Binney \& Tremaine 1987). Typical values for the ratio size/projected distance (which is an upper limit for the real size/distance) for our condensations range from $0.04(\mathrm{k} 2$ in IRAS $12112+0305)$ to 0.32 (R2 in IRAS $12112+0305)$, small enough to try to asses their stability against forces from the parent galaxy by means of tidal mass.

In general, the gravitational potential for a ULIRG is a complex function of the mass distribution of the system which evolves with time but, as a first approximation, it can be considered dominated by the masses of the main bodies of the 
interacting galaxies. We consider here two simple approaches: assuming that the TDG candidate is suffering the gravitational potential i) of a point mass in the mass centre and with the total mass of the system $\left(M_{\text {tid }}^{\mathrm{A}}\right)$ and ii) of the nearest galaxy $\left(M_{\text {tid }}^{\mathrm{B}}\right)$. The estimated values are shown in Table 4 . For $M_{\text {tid }}^{\mathrm{A}}$ we used $M=$ total mass of the system and $D=$ distance to the mass centre. Masses for the parent galaxies were taken from Colina et al. (2005) and García-Marín (2005). For the final tidal mass $\left(M_{\mathrm{tid}}\right)$ we considered the most conservative approach: the maximum of $M_{\text {tid }}^{\mathrm{A}}$ and $M_{\text {tid }}^{\mathrm{B}}$. We took into account that we are measuring the projected distance (always smaller than the real distance). Therefore, these values have to be seen as an upper limit to the real tidal mass.

An estimation of the dynamical mass of the candidates is needed to compare with the tidal masses. For those regions where a reliable measurement of the velocity dispersion was achieved, this can be done using:

$M\left(M_{\odot}\right)=$ const. $\times 10^{6} R_{\text {eff }}(\mathrm{kpc}) \sigma^{2}\left(\mathrm{~km} \mathrm{~s}^{-1}\right)$

Estimations of the constant range between 1.4 (Bender et al. 1992) and 2.2 (Terlevich \& Melnick 1981) depending on the method used for determination and assumed mass distribution. Here we adopted cte $=2.09$, a value derived from the Virial theorem, assuming a de Vaucouleurs profile (which describes quite well the luminosity distribution of spheroidal systems), that luminosity traces the mass of the system (Combes et al. 2002) and that the tridimensional velocity dispersion is related with the observed velocity dispersion as $\left\langle V^{2}\right\rangle=3 \sigma^{2}$ (McCrady et al. 2003). As it can be seen in last column of Table 4, most of the candidates with an estimation of the dynamical mass have a $M_{\text {dyn }} / M_{\text {tid }}$ ratio which supports the idea that most of the regions are stable against the forces exerted by the parent galaxy (mean $\sim 30$ ). Only R1 in IRAS $12112+0305$ and R2 in IRAS $16007+3743$ do not fulfil the criterion, but taking into account the uncertainties involved this is not very significant even for these two cases.

A last test could be a comparison between the relative velocity of a certain region and its escape velocity. This criterion cannot be used blindly. Firstly, it is a function of the distance which will always be larger than the projected one. Secondly, only one component of the velocity is being measured without having any information about the movements on the plane of the sky. Finally, since for a certain configuration it is not possible to determine if the region is closer or further from the observer than the mass centre, two possibilities for the relative movements between the region and the system are always possible. Due to all these uncertainties we cannot give to this comparison the same weight as the comparison between the dynamical and tidal masses. However, we found it useful to include it here for completeness, since used together with other indicators it can give us a better idea about the survival chances of the candidates.

In a simplified way, we consider that the gravitational potential is created by a point mass in the mass centre and with the total mass of the system. Relative and escape velocities can be compared looking at Cols. 7 and 8 of Table 4 . On average, the absolute values of the relative velocities are much smaller than predicted escape velocities $\left(189 \mathrm{~km} \mathrm{~s}^{-1}\right.$ vs. $346 \mathrm{~km} \mathrm{~s}^{-1}$ ). In order to take into account projection effects, we consider that a certain region will have good survival chances following this test if it satisfies the condition $\left|v_{\text {rel }}\right|-\left|v_{\text {esc }} \times \cos (\pi / 4)\right|>0$. According to this comparison, three out of twelve regions would pass this latter test.

\subsection{Comparison with models}

Although the observational characteristics of these condensations provide some hints that make us to consider them as putative TDG progenitors, a comparison with dynamical evolutionary models is needed to better predict if they will become independent objects.

The formation of TDG inside the tidal tails of interacting galaxies has been the subject of recent simulations. Wetzstein et al. (2005) showed the crucial role of the gas in the formation of these systems, contrary to the findings of Barnes \& Hernquist (1992). Bournaud \& Duc (2006) focused both the formation and evolution of these kinds of condensations. Typical masses of surviving ones are greater than $10^{8} M_{\odot}$. These simulations end $\sim 2$ Gyr after the beginning of the encounter. As an average $\sim 3.2$ condensations per galaxy are formed. From those, only the most massive $\left(\gtrsim 10^{9} M_{\odot}\right)$ and born at the tip of the tidal tail $\left(\sim 0.6-1.0 R_{\text {tail }}\right)$ survive the encounter 2 Gyr after the pericenter. We have performed a comparison of the properties derived for our condensations with those created in these simulations. Seven out of our twelve regions have more than $10^{9} M_{\odot}$. According to Bournaud \& Duc (2006), these have more chance to survive to the interaction process, losing only a small fraction of their mass. If the fate of our regions was similar to those of these condensations, most of them (with the exception of $\mathrm{R}$ in IRAS $15250+0305$ and R1 in IRAS $16007+3743$ ) would probably fall back to the parent galaxy losing part of its mass. A summary of the comparison with these simulations is shown in the sixth column of Table 5. One question that arises after this comparison is what is the origin of regions such as $\mathrm{R}$ in IRAS 14348-1447. Its observational properties indicate that it is a condensation of matter made out of the material of the interaction which is suffering a burst of star formation and hence, it should, in principle receive the same treatment as the other regions as possible entities able to survive to the merging process. However, to our knowledge, there is no simulation able to predict condensations of matter, even transient, outside the tidal tails.

How will they be evolving after 2 Gyrs? Unfortunately there are no evolutionary models covering this phase, for the range of masses of our regions. However, Kroupa (1997) and Metz \& Kroupa (2007) simulated the evolution of condensations with an initial mass of $10^{7} M_{\odot}$ (i.e. more than one order of magnitude lower than the present case) up to 10 Gyrs. They show that around 4-6 Gyr, depending on the model, the condensations retain $\sim 10 \%$ of their initial mass, and only $\sim 1 \%$ at $8-10$ Gyr. If these results could be scaled to our more massive regions, they would end up with masses of about $10^{7}-10^{8} M_{\odot}$. However, this extrapolation may be questionable and for a more firm conclusion we should wait for realistic simulations for the appropriate range of mass and time.

\subsection{Summary}

Due either to projection effects or to observational constrains, none of the tests performed above can alone tell us whether a certain region is going to survive as a future TDG or not. However, a region that satisfy most of the tests has more chances to survive.

We summarised the results of these tests in Table 5. We assigned a High probability of survival to those regions that satisfy all utilised criteria (i.e. only R of IRAS $15250+3609$ and R1 in IRAS 16007+3743), High-Medium and Medium probability to those that satisfy four and three of the utilised criteria respectively, and Low probability to those that satisfied only 
Table 5. Summary of the different TDG candidates survival criteria used.

\begin{tabular}{|c|c|c|c|c|c|c|}
\hline \multirow[t]{2}{*}{ Reg. } & \multicolumn{3}{|c|}{ Observational tests } & \multicolumn{2}{|c|}{ Comparison with simulations } & \multirow[t]{2}{*}{ Prob } \\
\hline & $L(\mathrm{H} \alpha)$ & $\sigma$ vs. $r$ & $M_{\text {tid }}$ vs. $M_{\text {cand }}$ & $M_{\mathrm{lim}}$ & Morph. & \\
\hline \multicolumn{7}{|c|}{ IRAS $08572+3915$} \\
\hline R1 & $\sqrt{ }$ & .. & $\ldots$ & $\ldots$ & $x$ & Low \\
\hline k7 & $\sqrt{ }$ & $\sqrt{ }$ & $\sqrt{ }$ & $x$ & $x$ & Medium \\
\hline \multicolumn{7}{|c|}{ IRAS $12112+0305$} \\
\hline R1 & $\sqrt{ }$ & $\sqrt{ }$ & $\sqrt{ }$ & $\sqrt{ }$ & ... & High-Medium \\
\hline R2 & $\sqrt{ }$ & $\sqrt{ }$ & $x$ & $\sqrt{ }$ & $\ldots$ & Medium \\
\hline $\mathrm{kc}$ & $\sqrt{ }$ & $\ldots$ & $\ldots$ & $\ldots$ & ? & Low \\
\hline $\mathrm{k} 1$ & $\sqrt{ }$ & $\ldots$ & $\ldots$ & $\ldots$ & $?$ & Low \\
\hline $\mathrm{k} 2$ & $\sqrt{ }$ & $\ldots$ & $\ldots$ & $\ldots$ & $?$ & Low \\
\hline \multicolumn{7}{|c|}{ IRAS 14348-1447 } \\
\hline $\mathrm{R}$ & $\sqrt{ }$ & $\sqrt{ }$ & $\sqrt{ }$ & $\sqrt{ }$ & $\ldots$ & High-Medium \\
\hline \multicolumn{7}{|c|}{ IRAS $15250+3609$} \\
\hline $\mathrm{R}$ & $\sqrt{ }$ & $\sqrt{ }$ & $\sqrt{ }$ & $\sqrt{ }$ & $\sqrt{ }$ & High \\
\hline \multicolumn{7}{|c|}{ IRAS $16007+3743$} \\
\hline R1 & $\sqrt{ }$ & $\sqrt{ }$ & $\sqrt{ }$ & $\sqrt{ }$ & $\sqrt{ }$ & High \\
\hline R2 & $\sqrt{ }$ & $\sqrt{ }$ & $x$ & $\sqrt{ }$ & $x$ & Low \\
\hline R3 & $\sqrt{ }$ & $\sqrt{ }$ & $\sqrt{ }$ & $\sqrt{ }$ & $x$ & High-Medium \\
\hline
\end{tabular}

The second column contains the luminosity criterion. The third column indicates when a certain condensation satisfies the relation velocity dispersion-radius of Terlevich \& Melnick (1981). The fourth column indicates if the system is stable against the tidal forces exerted by the parent galaxy(ies). The next column indicates when the estimated dynamical mass is above $10^{9} M_{\odot}$. The sixth column shows if a region is expected to survive according to the comparison with the simulation of Bournaud \& Duc (2006). The symbol $\sqrt{ }$ indicates that a certain condensation satisfies a criterion while $\times$ indicates that it fails. Symbols with a question mark are doubtful. The last column indicates the probability that a certain condensation become a TDG based on these criteria.

two or one. The most promising candidate is that detected in IRAS $15250+3609$. The relative large projected distance to the parent galaxy $(6.9 \mathrm{kpc})$, the advanced state of the merging process, its position in the $r-\sigma$ plane and the large velocity difference with relation to the parent galaxy (Colina et al. 2005; Monreal-Ibero 2004) make it the best candidate to survive the merging process. This region shows the way to be followed in the searching for such systems: a systematic search for regions with recent or ongoing star-formation among a sample of ULIRGs made out of galaxies in an advanced state of the merging process is appealing. The other region that should be mentioned here is R1 of IRAS $16007+3743$. Apparently it is at the tip of the tidal tail of a system that is starting its interaction and depending on its evolution it could finish in a region similar to that in IRAS $15250+3609$. In addition, there are three candidates with High-Medium probability (R1 of IRAS $12112+0305$, R of IRAS $14348-1447$ and R3 in IRAS $16007+3743)$. Two of them are new candidates while R in IRAS 1448-1447 had already been proposed as candidate by Mihos \& Bothun (1998). The continuity in the velocity field between them and their parent galaxy reduces the chance of survival.

The analysed systems are at higher redshift (and hence worst linear resolution) than their less luminous relatives previously studied. A follow-up of these candidates with IFS systems able to provide greater spatial resolution (i.e. Adaptive Optics assisted systems) would allow a search for independent velocity gradients in them, similar to the studies of Weilbacher et al. (2002) or Bournaud et al. (2004).

\section{Conclusions}

The present study is the first systematic attempt to try to establish TDG candidates among ULIRGs. Extranuclear star-forming regions identified as candidates to TDGs have been studied in a sample of low $-z$ ULIRGs on the basis of IFS and high angular resolution HST images.
As previously found in lower luminosity mergers, the present study shows that the presence of TDG candidates in ULIRGs is common. In particular we have identified 12 condensations in 5 ULIRGs (from an initial sample of 9), expanding the luminosity range of interacting galaxies and mergers showing this phenomenon.

We have characterised the main physical and kinematic properties of these candidates. In particular, their dynamical masses are in the $2 \times 10^{8}-1 \times 10^{10} M_{\odot}$ range with typical sizes of $\sim 750 \mathrm{pc}$. We found that most of these condensations follow the relation between effective radius and velocity dispersion of lower (globular clusters) and higher (elliptical) mass systems.

Starbursts have been identified within each TDG candidate. Their H II-like ionisation, relatively high metallicity $(12+$ $\log (\mathrm{O} / \mathrm{H}) \sim 8.6)$, and $\mathrm{H} \alpha$ equivalent widths $(34-257 \AA)$ are characteristic of young bursts of star formation (5-8 Myr) with stellar masses between $2 \times 10^{6}$ and $7 \times 10^{8} M_{\odot}$.

Using structural, physical and kinematical information, we have discussed the likelihood of survival for these candidates to internal as well as external forces on the basis of different dynamical tracers. Five out of the twelve initial regions present a High or High-Medium likelihood of survival as future TDG. We identified R in IRAS $15250+3609$ as our best candidate. It satisfies all our tests, occupies in the $r-\sigma$ plane a position close to dwarf elliptical galaxies, has relatively high relative velocity to the parent galaxy and has been found in a system in an advanced stage of the merging process.

Finally, we have outlined the working lines that need to be followed in the confirmation of these candidates as well as when looking for new ones M; i.e. IFS observation with high spatial resolution such as those provided by Adaptive Optics assisted IFS systems and the systematic search of external regions of star formation in ULIRGs in an advanced state of the merging process.

Acknowledgements. The authors wish to thank P. Weilbacher and L. M. Cairós as well as the referee, P.-A. Duc for useful comments and suggestions 
which helped to improve the paper. AMI acknowledges support from the Euro3D Research Training Network, funded by the EC (HPRN-CT-200200305). Financial support was provided by the Spanish Ministry for Education and Science through grant AYA2002-01055. Work based on observations with the William Herschel Telescope operated on the island of La Palma by the ING in the Spanish Observatorio del Roque de los Muchachos of the Instituto de Astrofísica de Canarias.

\section{References}

Alonso-Herrero, A., Rieke, G. H., Rieke, M. J., \& Scoville, N. Z. 2002, AJ, 124, 166

Amram, P., Mendes de Oliveira, C., Plana, H., et al. 2004, ApJ, 612, L5

Arribas, S., \& Colina, L. 2002, ApJ, 573, 576

Arribas, S., \& Colina, L. 2003, ApJ, 591, 791

Arribas, S., Carter, D., Cavaller, L., 1998 Proc. SPIE, 3355, 821

Arribas, S., Colina, L., \& Borne, K. D. 2000, ApJ, 545, 228

Arribas, S., Colina, L., \& Clements, D. 2001, ApJ, 560, 160

Arribas, S., Mediavilla, E., García-Lorenzo, B., del Burgo, C., \& Fuensalida, J. J. 1999, A\&AS, 136, 189

Baggett, S., et al. 2002, HST WFPC2 Data Handbook v. 4.0

Bastian, N., Emsellem, E., Kissler-Patig, M., \& Maraston, C. 2006, A\&A, 445, 471

Barnes, J. E. 2004, MNRAS, 350, 798

Barnes, J. E., \& Hernquist, L. 1992, Nature, 360, 715

Bekki, K., \& Shioya, Y. 2001, ApJS, 134, 241

Bender, R., Burstein, D., \& Faber, S. M. 1992, ApJ, 399, 462

Bingham, R. G., Gellatly, D. W., Jenkins, C. R., \& Worswick, S. P. 1994, Proc. SPIE, 2198, 56

Binney, J., \& Tremaine, S. 1987 (Princeton, NJ: Princeton University Press), 747 Blain, A. W., Smail, I., Ivison, R. J., Kneib, J.-P., \& Frayer, D. T. 2002, Phys. Rep., 369, 111

Borne, K. D., Bushouse, H, Lucas, R. A., \& Colina, L. 2000, ApJ, 529, 77

Braine, J., Duc, P.-A., Lisenfeld, U., et al. 2001, A\&A, 378, 51

Bournaud, F., \& Duc, P.-A. 2006, A\&A, 456, 481

Bournaud, F., Duc, P.-A., Amram, P., Combes, F., \& Gach, J.-L. 2004, A\&A, 425,813

Bushouse, H. A., Borne, K. D., Colina, L., et al. 2002, ApJS, 138, 1

Cairós, L. M., Caon, N., Papaderos, P., et al. 2003, ApJ, 593, 312

Caputi, K. I., Lagache G., Lin Y., et al. 2007, [arXiv: astro-ph/0701283]

Chapman, S. C., Windhorst, R., Odewahn, S., Yan, H., \& Conselice, C. 2003, ApJ, 599, 92

Clements, D. L., Sutherland, W. J., McMahon, R. G., \& Saunders, W. 1996, MNRAS, 279, 477

Colina, L., Arribas, S., \& Borne, K. D. 1999, ApJ, 527, L13

Colina, L., Arribas, S., Borne, K. D., \& Monreal, A. 2000, ApJ, 533, L9

Colina, L., Borne, K., Bushouse, H., et al. 2001, ApJ, 563, 546

Colina, L., Arribas, S., \& Monreal-Ibero, A. 2005, ApJ, 621, 725

Combes, F., Boisse, P., Mazure, A., Blanchard, A., \& Seymour, M. 2002, Galaxies and cosmology (2nd ed.). ed. F. Combes et al. (M. Seymour, Trans.) (New York: Springer)

Conselice, C. J., Chapman, S. C., \& Windhorst, R. A. 2003, ApJ, 596, L5 Cui, J., Xia, X.-Y., Deng, Z.-G., Mao, S., \& Zou, Z.-L. 2001, AJ, 122, 63

Denicoló, G., Terlevich, R., \& Terlevich, E. 2002, MNRAS, 330, 69

Duc, P.-A. \& Mirabel, I. F. 1994, A\&A, 289, 83

Duc, P.-A. \& Mirabel, I. F. 1998, A\&A, 333, 813

Duc, P.-A., Brinks, E., Springel, V., et al. 2000, AJ, 120, 1238

Duc, P.-A., Bournaud, F., \& Masset, F. 2004, A\&A, 427, 803

Edmunds, M. G., \& Pagel, B. E. J. 1984, MNRAS, 211, 507

Evans, A. S., Surace, J. A., \& Mazzarella, J. M. 2000, ApJ, 529, L85

Evans, A. S., Mazzarella, J. M., Surace, J. A., \& Sanders, D. B. 2002, ApJ, 580, 749

Filippenko, A. V. 1982, PASP, 94, 715

Gallagher, S. C., Charlton, J. C., Hunsberger, S. D., Zaritsky, D., \& Whitmore, B. C. 2001, AJ, 122, 163

García-Marín, M. (2005), DEA, Universidad Autónoma de Madrid

Geha, M., Guhathakurta, P., \& van der Marel, R. P. 2003, AJ, 126, 1794

Genzel, R., Tacconi, L. J., Rigopoulou, D., Lutz, D., \& Tecza, M. 2001, ApJ, 563,527

Goldader, J. D., Meurer, G., Heckman, T. M., et al. 2002, ApJ, 568, 651

Hibbard, J. E., van der Hulst, J. M., Barnes, J. E., \& Rich, R. M. 2001, AJ, 122, 2969

Howarth, I. D., \& Murray, J. 1988, DIPSO A Friendly Spectrum Analysis Program (Starlink User Note 50; Chilton: Rutherford Appleton Lab)
Hughes, D. H., Serjeant, S., Dunlop, J., et al. 1998, Nature, 394, 241 Iglesias-Páramo, J., \& Vílchez, J. M. 2001, ApJ, 550, 204 Iono, D., Yun, M. S., \& Mihos, J. C. 2004, ApJ, 616, 199

Ivison, R. J., Smail, I., Barger, A. J., et al. 2000, MNRAS, 315, 209 Kennicutt, R. C. 1984, ApJ, 287, 116

Kewley, L. J., Dopita, M. A., Sutherland, R. S., Heisler, C. A., \& Trevena, J. 2001, ApJ, 556, 121

Kim, D.-C., Veilleux, S., \& Sanders, D. B. 1998, ApJ, 508, 627

Knierman, K. A., Gallagher, S. C., Charlton, J. C., et al. 2003, AJ, 126, 1227

Kroupa, P. 1997, New Astron., 2, 139

Leitherer, C., Schaerer,D., Goldade, J. D., et al. 1999, ApJS, 123, 3

Lisenfeld, U., Braine, J., Duc, P.-A., et al. 2004, A\&A, 426, 471

López-Sánchez, Á. R., Esteban, C., \& Rodríguez, M. 2004, ApJS, 153, 243

Lonsdale, C., Farrah, D., \& Smith, H. 2006, [arXiv: astro-ph/0603031]

Marlowe, A. T., Meurer, G. R., Heckman, T. M., \& Schommer, R. 1997, ApJS, 112,285

Martini, P., \& Ho, L. C. 2004, ApJ, 610, 233

Mateo, M. L. 1998, ARA\&A, 36, 435

Mayya, Y. D. 1994, AJ, 108, 1276

McCrady, N., Gilbert, A. M., \& Graham, J. R. 2003, ApJ, 596, 240

Melo, V. P., Muñoz-Tuñón, C., Maíz-Apellániz, J., \& Tenorio-Tagle, G. 2005, ApJ, 619, 270

Mendes de Oliveira, C., Plana, H., Amram, P., Balkowski, C., \& Bolte, M. 2001, AJ, 121, 2524

Mendes de Oliveira, C., Cypriano, E. S., Sodré, L., Jr., \& Balkowski, C. 2004, ApJ, 605, L17

Metz, M., \& Kroupa, P. 2007, MNRAS, 376, 387

Meurer, G. R., Heckman, T. M., Leitherer, C., et al. 1995, AJ, 110, 2665

Mihos, J. C., \& Bothun, G. D. 1998, ApJ, 500, 619

Mihos, J. C., \& Hernquist, L. 1996, ApJ, 464, 641

Miller, J. S., \& Mathews, W. G. 1972, ApJ, 172, 593

Mirabel, I. F., Lutz, D., \& Maza, J. 1991, A\&A, 243, 367

Monreal-Ibero, A. 2004, Ph.D. Thesis, University of La Laguna

Monreal-Ibero, A., Arribas, S., \& Colina, L. 2006, ApJ, 637, 138

Moshir, M., et al. 1993, VizieR Online Data Catalog, 2156, 0

Mundell, C. G., James, P. A., Loiseau, N., Schinnerer, E., \& Forbes, D. A. 2004, ApJ, 614, 648

Origlia, L., \& Leitherer, C. 2000, AJ, 119, 2018

Pérez-González, P. G., et al. 2005, ApJ, 630, 82

Pope, A., Borys, C., Scott, D., et al. 2005, MNRAS, 358, 149

Pryor, C., \& Meylan, G. 1993, Structure and Dynamics of Globular Clusters, ASP Conf. Ser. 50, 357

Rieke, G. H., \& Lebofsky, M. J. 1985, ApJ, 288, 618

Sanders, D. B., \& Mirabel, I. F. 1996, ARA\&A, 34, 749

Sanders, D. B., Soifer, B. T., Elias, J. H., et al. 1988, ApJ, 325, 74

Schweizer, F., Miller, B. W., Whitmore, B. C., \& Fall, S. M. 1996, AJ, 112, 1839

Scoville, N. Z., Evans, A. S., Thompson, R., et al. 2000, AJ, 119, 991

Shioya, Y., Taniguchi, Y., \& Trentham, N. 2001, MNRAS, 321, 11

Smail, I., Ivison, R. J., \& Blain, A. W. 1997, ApJ, 490, L5

Smail, I., Chapman, S. C., Blain, A. W., \& Ivison, R. J. 2004, ApJ, 616, 71

Surace, J. A., \& Sanders, D. B. 1999, ApJ, 512, 162

Surace, J. A., \& Sanders, D. B. 2000, AJ, 120, 604

Tacconi, L. J., Neri, R., Chapman, S. C., et al. 2006, ApJ, 640, 228

Tacconi, L. J., Genzel, R., Lutz, D., et al. 2002, ApJ, 580, 73

Terlevich, R., \& Melnick, J. 1981, MNRAS, 195, 839

Temporin, S., Weinberger, R., Galaz, G., \& Kerber, F. 2003, ApJ, 587, 660

Torres-Peimbert, S., Peimbert, M., \& Fierro, J. 1989, ApJ, 345, 186

Trager, S. C., Djorgovski, S., \& King, I. R. 1993, Structure and Dynamics of Globular Clusters, ASP Conf. Ser., 50, 347

Veilleux, S., \& Osterbrock, D. E. 1987, ApJS, 63, 295

Veilleux, S., Sanders, D. B., \& Kim, D.-C. 1999, ApJ, 522, 139

Veilleux, S., Kim, D.-C., \& Sanders, D. B. 2002, ApJS, 143, 315

Vílchez, J. M., \& Esteban, C. 1996, MNRAS, 280, 720

Weilbacher, P. M., Duc, P.-A., Fritze v. Alvensleben, U., Martin, P., \& Fricke, K. J. 2000, A\&A, 358, 819

Weilbacher, P. M., Fritze-v. Alvensleben, U., Duc, P.-A., \& Fricke, K. J. 2002 , ApJ, 579, L79

Weilbacher, P. M., Duc, P.-A., \& Fritze-v. Alvensleben, U. 2003, A\&A, 397, 545

Wetzstein, M., Naab, T., \& Burkert, A. 2007, MNRAS, 375, 805

Whitford, A. E. 1958, AJ, 63, 201

Whitmore, B. C., Zhang, Q., Leitherer, C., et al. 1999, AJ, 118, 1551

Zepf, S. E., Ashman, K. M., English, J., Freeman, K. C., \& Sharples, R. M. 1999,

AJ, 118,752

Zwicky, F. 1956, Ergeb. Exakt. Naturwiss, 29, 344 